\title{
Adenovirus-mediated TRAIL expression and downregulation of Bcl-2 expression suppresses non-small cell lung cancer growth in vitro and in vivo
}

\author{
HAIPING ZHANG ${ }^{1}$, AIHUA SUI ${ }^{2}$, ZHENLI WANG $^{3}$, SHIHAI LIU ${ }^{2}$ and RUYONG YAO ${ }^{1,2}$ \\ ${ }^{1}$ Department of Clinical Laboratory and ${ }^{2}$ Central Laboratory, The Affiliated Hospital of Qingdao University Medical \\ College, Qingdao 266003; ${ }^{3}$ Department of Pharmacology, The First Sanatorium of Jinan \\ Military District, Qingdao 266001, P.R. China
}

Received February 17, 2012; Accepted April 20, 2012

DOI: $10.3892 / \mathrm{ijmm} .2012 .998$

\begin{abstract}
Primary or acquired resistance to current treatment methods remains a major factor in clinical oncology and may be caused by failures in apoptosis programs. TNF-related apoptosis-inducing ligand (TRAIL) can induce apoptosis in several human cancer cell types. However, not all cancer cells are susceptible to TRAIL and mechanisms of resistance and new strategies to enhance sensitivity are an area of intense investigation. Therefore, we investigated whether TRAIL resistance is due to $\mathrm{Bcl}-2$ levels. In this study, we generated an adenoviral vector, Ad5.TRAIL/siBcl2, that permitted co-expression of shRNA against Bcl-2 and the tumor necrosis factor-related apoptosis-inducing ligand (TRAIL) therapeutic gene from a cytomegalovirus promoter. Infection with Ad5. TRAIL/siBcl2 resulted in significant cytotoxicity in non-small cell lung cancer (NSCLC) cells in vitro. In contrast, it had no effect on a normal lung cell line, WI-38. Impressively, treatment of the established NSCLC tumor model with Ad5.TRAIL/ siBcl2 resulted in significant tumor regression, compared with other adenoviruses. This potent antitumor activity induced by Ad5.TRAIL/siBcl2 was due to strong inhibition of Bcl-2 and high expression of TRAIL. Thus, this study may provide a framework for future clinical applications of Ad5.TRAIL/ siBcl2 in lung tumor gene therapy.
\end{abstract}

\section{Introduction}

Lung cancer is the leading cause of cancer death throughout the world, causing $\sim 1.2$ million deaths annually and an estimated >250,000 deaths in China during 2010 (1,2). Lung

Correspondence to: Professor Ruyong Yao, Central Laboratory, The Affiliated Hospital of Qingdao University Medical College, 16 Jiangsu Road, Qingdao 266003, P.R. China

E-mail:yry0303@yahoo.com.cn

Key words: tumor necrosis factor-related apoptosis-inducing ligand, B-cell lymphoma 2, adenovirus, non-small cell lung cancer, apoptosis cancer is clinically divided into two subtypes, small cell lung cancer (SCLC) and non-small cell lung cancer (NSCLC). The latter is the most common type, accounting for $85-90 \%$ of total cases (3). Although recent studies have shown that adjuvant chemotherapy improves survival in completely resected NSCLC, only $5-15 \%$ of treated individuals ultimately benefit (4). Resistance of NSCLC to current treatment protocols still remains a major concern in cancer therapy (5).

Tumor necrosis factor-related apoptosis-inducing ligand (TRAIL) is a highly promising anticancer agent with pronounced pro-apoptotic activity towards various malignant cell types, including lung cancer. Importantly, TRAIL essentially lacks activity towards normal cells (6). Based on these characteristics, recombinant soluble TRAIL (sTRAIL) preparations have recently entered clinical trials, with promising preliminary studies on anti-tumor activity and safety (7). TRAIL engages its receptors via the extrinsic apoptotic pathway and recruits caspase- 8 , which is then cleaved to its active form. Activated caspase- 8 then cleaves the BH3-only molecule, Bid, which then interacts with mitochondrial anti- and pro-apoptotic molecules. Previous studies have demonstrated that TRAIL modulates the apoptotic response by binding to receptors, including the death receptor 4 (DR4, TRAIL-R1) (8), KILLER/DR5 (TRAIL-R2) (9), TRID (DcR1, TRAIL-R3) (10), TRUNDD (DcR2, TRAIL-R4), and osteoprotegerin (OPG) (11). Both DR4 and DR5 have a C-terminal death domain that signals through downstream caspase activation. However, it is not yet fully understood how TRAIL induces tumor-cell apoptosis. Hence, a great deal of research has been focused on improving the potency of TRAIL-based therapeutic approaches, some by establishing new modalities for combined treatment with TRAIL and other agents. Bcl-2 (B-cell lymphoma 2) is the founding member of the Bcl-2 family of apoptosis regulator proteins encoded by the $\mathrm{Bcl}-2$ gene. $\mathrm{Bcl}-2$ is highly expressed in many cell lines and primary tumors including lung tumors and its expression has been associated with drug resistance $(12,13)$. Thus, novel strategies are necessary to target resistance of tumors with Bcl-2 overexpression.

In this study, focusing on the lung cancer line A549, we constructed a recombinant adenovirus Ad5.TRAIL/siBcl2, 
with overexpression of TRAIL and downregulation of Bcl-2. We investigated the therapeutic gene of Ad5.TRAIL/siBcl2 and explored its antitumor potential in experimental lung tumors.

\section{Materials and methods}

Cell culture and reagents. The human embryonic kidney cell line HEK293, the human lung tumor cell lines A549 and HCC827 as well as the normal lung tissue cell line WI-38, were obtained from the American Type Culture Collection (Rockville, MD) and cultured in Dulbecco's modified Eagle's medium (DMEM) supplemented with $10 \% \mathrm{FBS}$ and $100 \mathrm{IU} / \mathrm{ml}$ penicillin/ streptomycin in a $37^{\circ} \mathrm{C}$ humidified incubator with $5 \% \mathrm{CO}_{2}$. The recombinant adenovector ${ }^{\mathrm{TM}}$ system was purchased from Stratagene (Carlsbad, CA) for the construction of adenoviruses. The anti-TRAIL, the Bcl-2 and the HRP-conjugated anti-mouse IgG antibodies were obtained from Santa Cruz Biotechnology, Inc. (Santa Cruz, CA).

MTT assay. The cytotoxic activity of TRAIL protein was determined based on cycotoxicity to the human lung tumor cell line A549, using an MTT assay. Briefly, cells were seeded in 96-well tissue culture plates at a density of $5 \times 10^{3}$ cells/well and then treated at the concentration of $0,1,10$ and $100 \mathrm{ng} / \mathrm{ml}$ TRAIL protein in the medium. The following day, the medium was removed and $100 \mu \mathrm{l}$ of fresh medium containing $0.5 \mathrm{mg} / \mathrm{ml}$ MTT (Roche Diagnostics GmbH; Mannheim, Germany) was added to each well. The cells were incubated at $37^{\circ} \mathrm{C}$ in a humidified atmosphere with $5 \% \mathrm{CO}_{2}$ for $4 \mathrm{~h}$, followed by the addition of $150 \mu \mathrm{l}$ of solubilization solution $[0.01 \mathrm{~mol} / 1 \mathrm{HCl}$ in $100 \mathrm{~g} / \mathrm{l}$ sodium dodecyl (SDS)] to each well and the incubation of cells for further $10 \mathrm{~min}$ at $37^{\circ} \mathrm{C}$ with gentle shaking. The optical density of the plates was measured using the spectrophotometrical absorbance at $570 \mathrm{~nm}$ in the Microplate Reader Model 550 (Bio-Rad, Hercules, CA).

Viability and caspase-3/7 assays. For the Apo-One ${ }^{\circledR}$ Homogeneous caspase-3/7 assay (Promega Corporation, Madison, WI), cells were seeded into 96-well plates at $2 \times 10^{4}$ cells/well, incubated overnight and subsequently treated with TRAIL (Alexis Corporation, Lausanne, Switzerland). The substrate for the caspase assay was added after 0-24 h of treatment (as indicated in the text) and plates were read $2 \mathrm{~h}$ later using a Tecan Plate Reader (Tecan, Group. Ltd., Switzerland). All treatments were done in triplicate. Background absorbance was determined by incubating media with substrate alone and subtracting the values from wells containing cells.

Preparation of cell extracts and western blot analysis. Protein lysates were prepared on ice in RIPA buffer $[50 \mathrm{mM}$ Tris-HCl (pH 8.0), $150 \mathrm{mM} \mathrm{NaCl}, 0.1 \%$ SDS, $1 \% \mathrm{NP} 40$ and $0.5 \%$ sodium deoxycholate] with freshly added $0.1 \mathrm{mg} / \mathrm{ml}$ phenylmethylsulfonyl fluoride, $1 \mathrm{~mm}$ sodium orthovanadate and $1 \mathrm{mg} / \mathrm{ml}$ aprotinin. Protein concentrations were determined using the Bio-Rad protein assay system (Bio-Rad Laboratories, Inc., Richmond, CA). Aliquots of cell extracts containing 20-50 mg of total protein were resolved in 12\% SDS-PAGE and transferred to $0.45 \mathrm{~mm}$ nitrocellulose membranes (Osmonics, Westborough, MA). Filters were blocked for $1 \mathrm{~h}$ at room temperature (RT) in Blotto A [5\% nonfat milk powder in TBS-T: $10 \mathrm{~mm}$ Tris- $\mathrm{HCl}$ (pH 8.0), $150 \mathrm{~mm} \mathrm{NaCl}, 0.05 \%$ Tween-20], and then incubated for $1 \mathrm{~h}$ at room temperature in Blotto A containing a 1:1,000 dilution of rabbit anti-TRAIL, anti-Bcl-2 and anti-GAPDH monoclonal antibodies. After washing in TBS-T buffer ( $5 \mathrm{~min}$ at RT), filters were incubated for $1 \mathrm{~h}$ at room temperature in Blotto A containing a 1:10,000 dilution of peroxidase conjugated anti-rabbit or anti-mouse secondary antibody (Amersham Life Science, Arlington Heights, IL). After washing in TBS-T, ECL was performed according to the manufacturer's instructions. All results were normalized to GAPDH protein expression.

Construction of gene silencing and adenoviral vectors. Plasmid pGenesil-1 (Wuhan Genesil Biotechnology Co., Ltd., Wuhan, China) was used to express small hairpin RNA (shRNA) targeting human Bcl-2. The following hairpin inserts were used, Bcl2-1F, 5'-GATCCGGTACGATAACCGGGAGATA GTTTCAAGACGACTATCTCCCGGTTATCGTACTTTTT TA-3' and Bcl2-1R, 5'-AGCTTAAAAAAGTACGATAAC CGGGAGATAGTCGTCTTGAAACTATCTCCCGGTTATC GTAC-3'; Bcl2-2F, 5'-GATCCGGGAGGATTGTGGCCTTC TTTTTCAAGACGAAAGAAGGCCACAATCCTCCTTTT TTA-3' and Bcl2-2R: 5'-AGCTTAAAAAAGGAGGATTG TGGCCTTCTTTCGTCTTGAAAAAGAAGGCCACAATC CTCCCG-3'; shRNA control, Con-F, 5'-GATCCGCCACTTG GACCAGTATTATTTCAAGACGATAATACTGGTCCAAG TGGTTTTTTA-3' and Con-R, 5'-AGCTTAAAAAACCACT TGGACCAGTATTATCGTCTTGAAATAATACTGGTCCA AGTGGCG-3'. The insert was cloned into BamHI and HindIII site, which is downstream of the human U6 promoter (hU6), an RNA polymerase III promoter. All the constructions were confirmed by DNA sequencing. The correct plasmid, named pGenesil-Bcl2-1, pGenesil-Bcl2-2 and pGenesil-con, was used for screening the silence genes.

The sense primer (5'-GAAGATCTGTGAGAGAAAGAGG TCCTCAGAGA-3') and the antisense primer (5'-GGGGTA CCTTAGCCAACTA AAAAGGCCCCGA-3') were used for cloning the human TRAIL gene directly from plate purchased from Sino Biological, Inc. (Beijing, China) by using the polymerase chain reaction (PCR). The cloned TRAIL cDNA fragment was ligated into the pShuttle-CMV vector to form pShuttle-CMV/TRAIL for expressing TRAIL. The pShuttleCMV/TRAIL, which was cut by SalI and HindIII, was ligased with the fragment hU6-Bcl2-2 from pGenesil-Bcl2-2. The recombinant pAdeasy-1 backbone vector and pShuttle-CMV/ TRAIL, pShuttle-CMV/siBcl2 or pShuttle-CMV/TRAIL/ siBcl2 vector, DNA linealized with PmeI digestion, were further co-transfected into the bacteria BJ5183 cells. The resultant pAd5.TRAIL, pAd5.siBcl2 and pAd5.TRAIL/siBcl2 plasmid vectors were purified from the above transfected BJ5183 cells, then linealized by $\mathrm{PacI}$ digestion and then transfected into the HEK293 cells by lipofectamine 2000 (Invitrogen Life Technologies, Carlsbad, CA), leading to the formation of the recombinant adenoviruses Ad5.siBcl2, Ad5.TRAIL and Ad5. TRAIL/siBcl2. The adenoviruses were amplified in HEK293 cells, purified by cesium chloride ultracentrifugation and stored at $-80^{\circ} \mathrm{C}$ before use. Titers were determined by plaque assay on HEK 293 cells.

Apoptotic cell assay. Cells seeded in 96-well tissue culture plates were treated with PBS, Ad5.eGFP, Ad5.siBcl2, Ad5.TRAIL or 

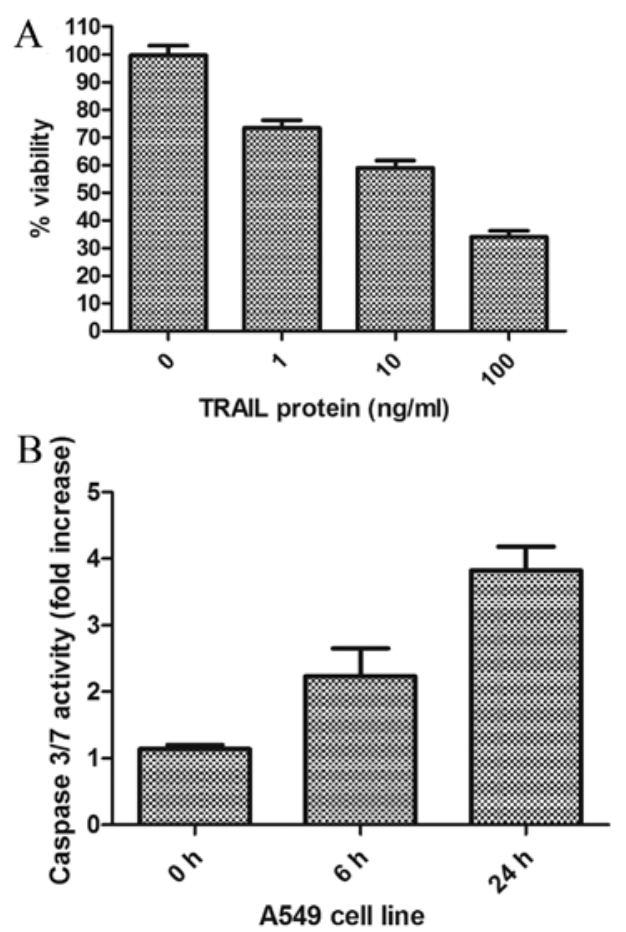

Figure 1. Comparison of TRAIL sensitivity in A549 cells. (A) Viability was measured $24 \mathrm{~h}$ after exposure to different concentrations of TRAIL. (B) Caspase-3/7 activity was determined after incubation with $100 \mathrm{ng} / \mathrm{ml}$ TRAIL. Similar results were obtained in at least 3 independent experiments. Bars are the mean $\pm \mathrm{SD}$ (from 3 or 6 experiments).

Ad5.TRAIL/siBcl2. After $48 \mathrm{~h}$ of treatment, cells were incubated with MTT and followed by the MTT assay method.

Tumor studies. Athymic nude and BalB/C mice were obtained from the Shanghai Experimental Animal Center (Shanghai, China) and maintained according to the Animal Research Committee's guidelines at Qingdao University (Qingdao, China). Briefly, a total of $8 \times 10^{6}$ A549 cells were injected into the left dorsal flank of mice (10 animals for each treatment) and then monitored every other day for tumor growth. When tumors reached $40-50 \mathrm{~mm}^{3}$, animals were randomly divided and received Ad5.eGFP, Ad5.siBcl2, Ad5.TRAIL or Ad5. TRAIL/siBcl2 treatment. All treatments were given every other day for a total of 5 doses. Tumor growth was monitored by measuring perpendicular tumor diameters using an electronic digital caliper. Tumor volumes were calculated by the formula: tumor size $=a b^{2} / 2$, where $a$ is the largest and $b$ is the smallest of the 2 dimensions. The tumor-bearing mice were sacrificed 10 days after injection with different viruses and the tumors were removed, weighed and examined by TUNEL assay.

TUNEL assay. In situ cell apoptosis of tumor sections taken on Day 10 post-treatment was analyzed by using a commercially available TUNEL kit (Promega Corporation). Briefly, tumor sections were fixed with a freshly prepared paraformaldehyde solution (4\% in PBS, pH 7.4). After 30 min of incubation at room temperature, cells were washed with PBS and resuspended in a permeabilization solution $(0.1 \%$ Triton X-100 in $0.1 \%$ sodium citrate) for $2 \mathrm{~min}$ on ice. After an additional wash with PBS, cells were labeled using the TUNEL kit according
A

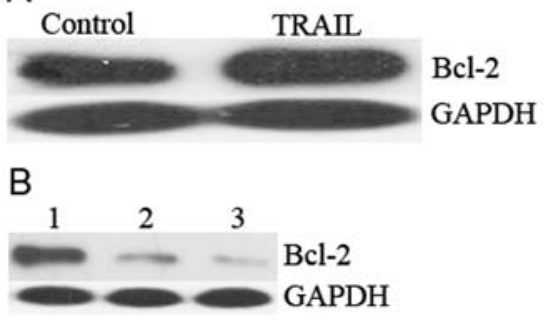

Figure 2. Effect of Bcl-2 overexpression on TRAIL-induced apoptosis. (A) Effect of Bcl-2 overexpression on TRAIL-induced apoptosis in lung tumor cell line A549. (B) Efficient Bcl-2 protein by shRNA. A549 cells were transfected with control (pGenesil-con) or Bcl-2 (pGenesil-Bcl2-1 and pGenesil-Bcl2-2). Bcl-2 protein levels were assessed $48 \mathrm{~h}$ later by immunoblotting. 1, pGenesil-con was transfected into A549 cells; 2, pGenesil-Bcl2-1 was transfected into A549 cells; 3, pGenesil-Bc12-2 was transfected into A549 cells; GAPDH was loading as control.

to the manufacturer's instructions. The apoptotic index was calculated by the formula: apoptotic index $=$ (total number of apoptotic cells/total number of cells) x $100 \%$.

Statistical analysis. Student's t-test and One-way ANOVA analysis were performed for continuous variables. The $\chi^{2}$ test or Fisher's exact test were used for categorical variables. The error bars represent the standard error of the mean. Statistical significance for all the tests, assessed by calculating P-value, was $<0.05$ from two-sided tests. The statistical analyses were performed using SAS 9.0 software (SAS Institute, Inc., Cary, NC).

\section{Results}

The susceptibility of A549 lung cancer cell lines to TRAIL protein. The goal of this study was to determine the sensitivity of lung cancer cells to TRAIL protein treatment. A549, the common NSCLC cell line, was used for this purpose. As shown in Fig. 1A, exposure of A549 cells to increasing concentrations of recombinant TRAIL protein resulted in a dose-dependent decrease in viability. To confirm that the response to TRAIL was apoptotic, we performed caspase-3/7 activity assays (Fig. 1B). In A549 cells, a 2- to 3-fold increase in caspase activity was measured at 6 and $24 \mathrm{~h}$ respectively, following exposure to TRAIL protein. This level of caspase activation may be sufficient to cause cell death. However, there was some resistance to the TRAIL induced apoptosis. TRAIL resistance in the cancer cells was due to differences in expression of various proteins of the apoptotic pathway, including TRAIL receptors, cFLIP, Bax, Bcl-2, caspase-3 or X-linked mammalian inhibitor of apoptosis protein (14). To explore whether Bcl-2 presents a main resistance point in A549 cells, we analyzed Bcl-2 expression with or without TRAIL treated cells. As presented in Fig. 2A, enhanced Bcl-2 expression was observed in TRAIL treatment cells on the concentration of $100 \mathrm{ng} / \mathrm{ml}$. Because Bcl-2 has been implicated in apoptotic regulation in other organisms, we determined whether Bcl-2 deficiency affected the in vitro apoptosis of mammalian cells using 'knockdown' (KD) technology. Two independent small hairpin RNA (shRNA) specific for human Bcl-2 (siBcl2-1 and siBcl2-2) and a control (siCNT) were introduced into the 
A

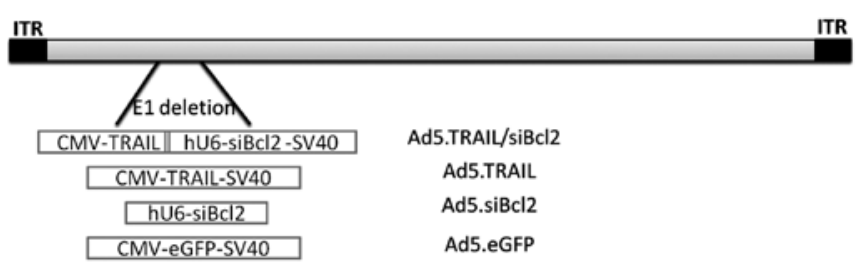

B

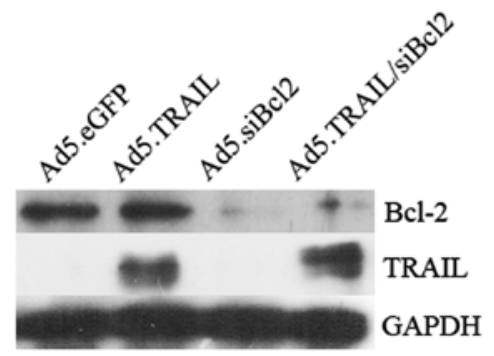

Figure 3. Construction and characterization of the adenoviral vectors co-expressing both shRNA and transgene. (A) Cartoon of recombinant adenovirus vector. The vector has an expression cassette containing a CMV promoter and/or human U6 promoter for expression target genes. (B) Expression of Bcl-2 and TRAIL in A549 cells after infection with adenoviruses. A549 cells were infected with Ad5.eGFP, Ad5.siBcl2, Ad5.TRAIL or Ad5.TRAIL/siBcl2 at MOI of 10 . After $48 \mathrm{~h}$ the cells were collected for Bcl-2 and TRAIL determination by western blot analysis.

A549 cell line to verify their efficiency. After $48 \mathrm{~h}$, the level of $\mathrm{Bcl}-2$ protein was reduced to $<10 \%$ of the control in A549 cells transfected with siBcl2-1 or siBcl2-2, whereas introduction of siCNT had no effect on Bcl-2 protein levels (Fig. 2B). To test the apoptotic efficiency, siBcl2-2 was chosen for the downstream experiment in vitro and in vivo.

Characterization of adenoviral vectors for co-expression of shRNA and transgene in vitro. To achieve maximum efficiency of Bcl-2 silencing and TRAIL expression in lung cancer cells, adenoviral vectors (Ad5.eGFP, Ad5.shBcl2, Ad5.TRAIL and Ad5.TRAIL/siBcl2) were generated for co-expressing shRNA and TRAIL (Fig. 3A). To compare the efficiency of shRNAmediated silencing of Bcl-2 and expression of TRAIL, we infected A549 cells with 4 adenoviral constructs and the expression of Bcl-2 and TRAIL was measured. As shown in Fig. 3B, the level of Bcl-2 expression was comparable in the cells infected with Ad5.eGFP, Ad5.TRAIL and cells without infection. In contrast, infection with Ad5.siBcl2 and Ad5.TRAIL/siBcl2 resulted in significant reduction of $\mathrm{Bcl}-2$ gene expression. The expression of Bcl-2 was almost undetectable in cells infected with Ad5.siBcl2 and Ad5.TRAIL/siBcl2 (Fig. 3B). The high and comparable level of TRAIL expression was only observed in the cells infected with Ad5.TRAIL and Ad5.TRAIL/siBcl2. This indicated that Ad5.TRAIL/siBcl 2 could induce efficient silencing of Bcl-2 and expression of TRAIL.

Ad5.TRAIL/siBcl2 induces tumor-cell cytotoxicity in vitro. To evaluate whether Ad5.TRAIL/siBcl 2 could impede lung cancer cell proliferation, we performed cell viability assay in the lung cancer cells after infection with adenoviral vectors. As shown in Fig. 4, both Ad5.TRAIL and Ad5.siBcl2 could reduce cell viability in lung cancer cells. However, there was significant
A549

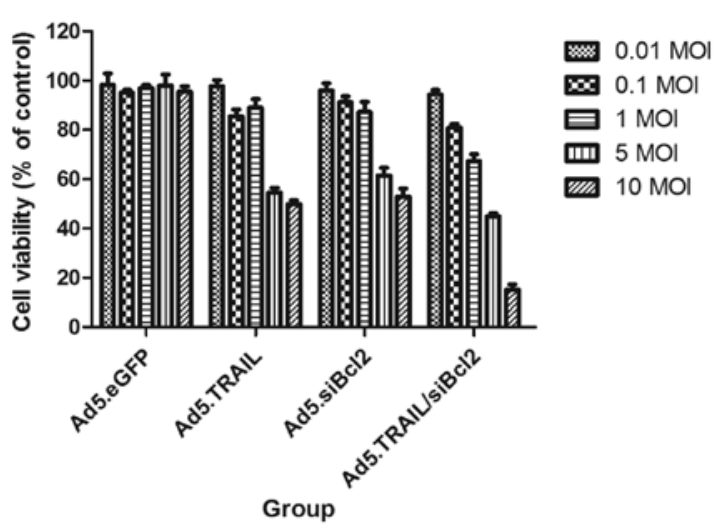

HCC827

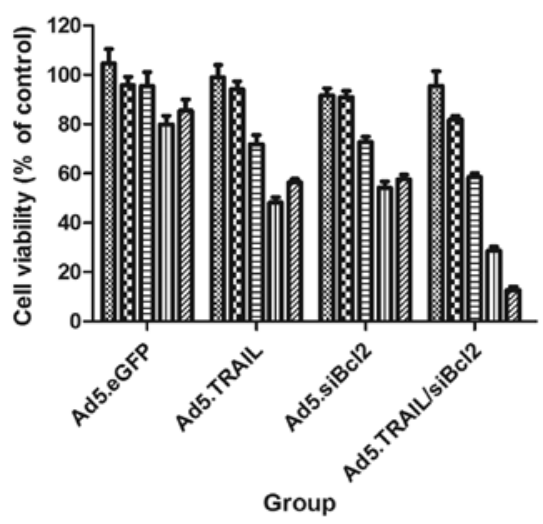

$0.01 \mathrm{MOI}$

$0.1 \mathrm{MO}$

吕 $1 \mathrm{MO}$

血 $5 \mathrm{MOI}$

때 $10 \mathrm{MOI}$

Wh-38

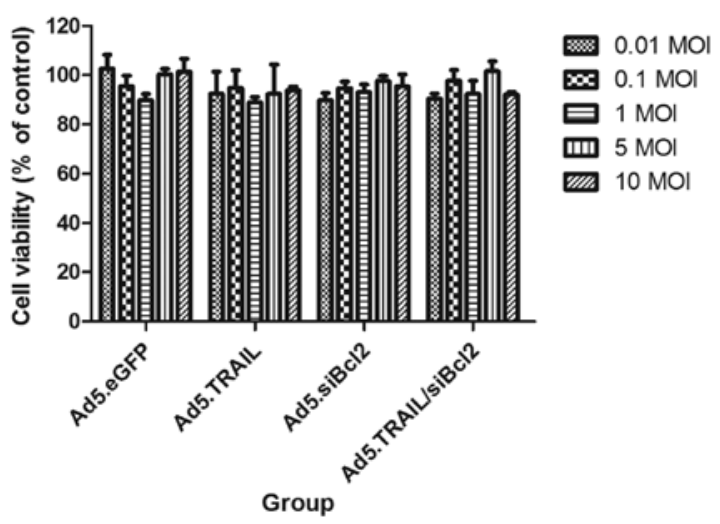

Figure 4. Induction of cytotoxicity by adenoviral vectors in vitro. The lung tumor cells (A549, HCC827) and the normal cells (WI-38) were infected with Ad5.eGFP, Ad5.siBcl2, Ad5.TRAIL or Ad5.TRAIL/siBcl2. Cell viability was determined by MTT assay at different timepoints after infection. Cell viability was calculated as a percentage with respect to cells without adenovirus infection. The data are presented as the mean $\pm \mathrm{SD}$ of 3 independent experiments. ${ }^{*} \mathrm{P}<0.01$, compared with either Ad5.eGFP, Ad5.siBcl2 or Ad5.TRAIL.

reduction in cell viability in tumor cells infected with Ad5. TRAIL/siBcl2, comparing to cells infected with Ad5.TRAIL and $\mathrm{Ad} 5 . \mathrm{shBcl} 2$. These results suggested that Bcl-2 silencing sensitized lung cancer cells to TRAIL. In contrast, Ad5.siBcl2, Ad5.TRAIL or Ad5.TRAIL/siBcl2 exhibited low cytotoxicity on normal lung cells WI-38. These data indicated that Ad5. TRAIL/siBcl2 had enhanced antitumor activity in lung tumor cells and maintained low toxicity to normal lung cells.

To investigate the mechanism of apoptosis, we analyzed A549 cells infected with Ad5.eGFP, Ad5.TRAIL, Ad5.shBcl2 
A
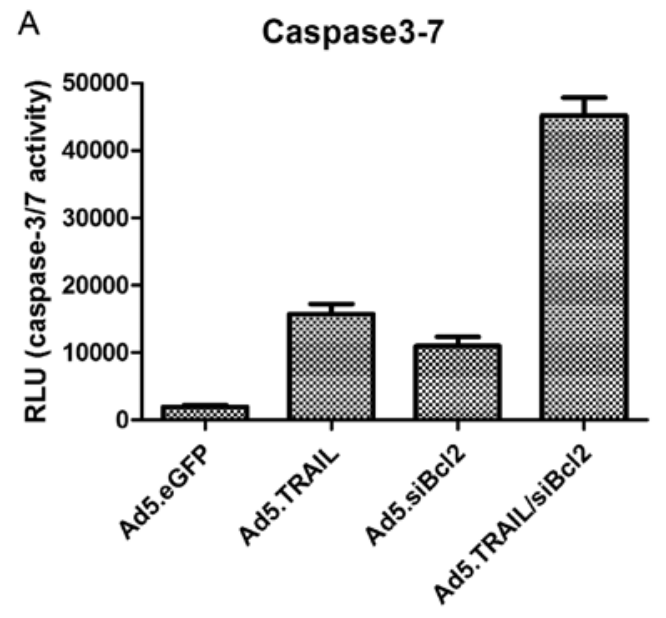

B

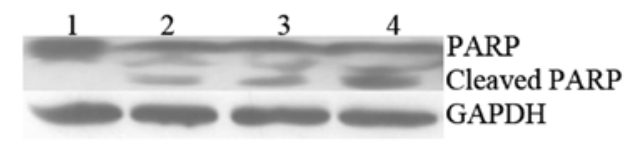

Figure 5. Recombinant adenoviruses induced caspase-3/7 activity and cleavage of PARP in NSCLC cells. (A) Caspase-3/7 assay analyses for caspase-3 expression in A549 cells infected with Ad5.eGFP, Ad5.siBcl2, Ad5.TRAIL or Ad5.TRAIL/siBcl2 (100 pfu/cell) for $48 \mathrm{~h}$. Columns, mean of 3 independent experiments. (B) A549 cells, infected with recombinant adenoviruses for $48 \mathrm{~h}$, were washed twice with cold PBS and lysed on ice in cold lysis buffer. Cell lysates were subjected to western blot analysis using PARP antibodies, followed by HRP-conjugated secondary antibody. Blots were developed using enhanced chemiluminescence system. Cleavage of the DNA repair enzyme, PARP, to its inactive $85 \mathrm{kDa}$ fragment was obviously observed in 3 cell types. No PARP cleavage was observed in cells treated with Ad5.eGFP.

or Ad5.TRAIL/siBcl2 for $48 \mathrm{~h}$ by caspase-3/7 activity assay and western blot analysis. Caspase-3/7 activity was increased in the presence of Ad5.TRAIL/siBcl2 infection, indicating that TRAIL plus siBcl-2 could sensitize NSCLC cells to the cytotoxic actions (Fig. 5A). The analysis of infected A549 cancer cells demonstrated the activation of poly(ADP-ribose) polymerase (PARP) after infection with different adenoviruses. The cleaved forms of PARP increased and the uncleaved form of PARP decreased, which indicated the activation of caspase cascade after treatment with adenoviruses. The most obvious effect of caspase activation was observed in the cells treated with Ad5.TRAIL/siBcl2 (Fig. 5B). These results suggest that the combined use of TRAIL and siBcl-2 can induce the caspase cascade activation in a synergistic manner.

Ad5.TRAIL/siBcl2 suppresses tumor growth in vivo. To explore the in vivo antitumor effect of adenoviruses, the athymic nude mice (10 mice/group), bearing A549 tumors $\left(0.2-0.3 \mathrm{~cm}^{3}\right)$ were intrathecally (i.t.) injected with Ad5.eGFP, Ad5.siBcl2, Ad5. TRAIL and Ad5.TRAIL/siBcl2 $\left(1 \times 10^{7} \mathrm{pfu} / 50 \mu \mathrm{l}\right)$. The tumor growth was monitored daily. As shown in Fig. 6, the tumor growth was significantly decreased in the groups of mice treated with Ad5.TRAIL/siBcl2, compared with the tumor growth of mice treated with Ad5.GFP $(\mathrm{P}<0.01)$, indicating that Ad5.TRAIL/siBcl2 can induce efficient tumor growth suppression in vivo. To understand the mechanism of tumor inhibition, tumor sections were analyzed for apoptosis by TUNEL assay. As shown in Fig. 7, tumors from the mice injected with Ad5.
Tumor volume

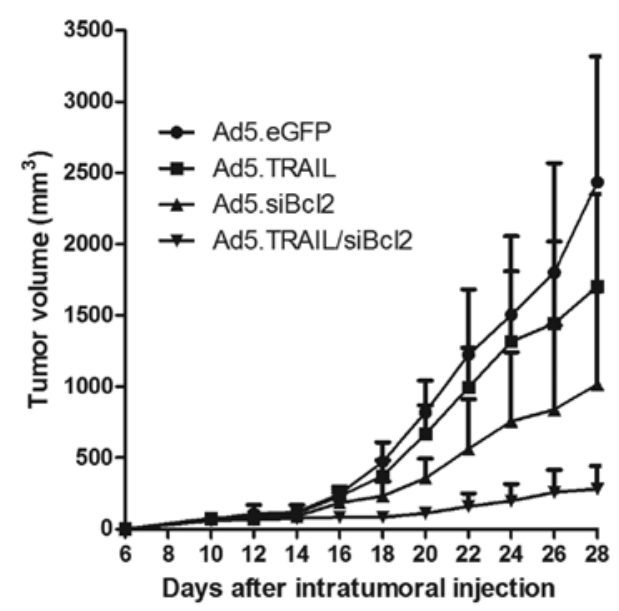

Figure 6. Antitumor activity by adenoviral vectors in established lung tumor model. Tumors were established in nude mice by implantation of A549 cells. When the tumors became $40-50 \mathrm{~mm}^{3}$, the animals were treated with an intratumor injection of Ad5.eGFP, Ad5.siBcl2, Ad5.TRAIL or Ad5.TRAIL/ siBcl2. The tumor size was measured and the tumor volume was calculated as follows: length $\mathrm{x}$ width ${ }^{2} / 2$. The data are expressed as the means of the tumor volume $\pm \mathrm{SD}(\mathrm{n}=8)$. ${ }^{*} \mathrm{P}<0.01$, compared with either Ad5.eGFP, Ad5. siBcl2 or Ad5.TRAIL.

TRAIL/siBcl2 demonstrated extensive apoptosis $26.24 \pm 2.62 \%$, compared with the group treated with Ad5.eGFP, Ad5.siBcl2 or Ad5.TRAIL. The apoptotic index, expressed as the average percentages of TUNEL-positive cells from 10 random visual fields, were $1.94 \pm 0.53,7.25 \pm 1.42$ and $13.15 \pm 2.38 \%$ in Ad5. eGFP, Ad5.siBcl2 and Ad5.TRAIL, respectively.

\section{Discussion}

Despite the aggressive treatment regimens, patients with diagnosis of NSCLC still have a poor prognosis (15). Current attempts to improve the survival of these patients largely depend on novel strategies designed to overcome the intrinsic resistance of NSCLC to the established therapies $(16,17)$. The idea to directly trigger apoptosis in cancer cells by stimulating death receptors at the cell surface has gained considerable attention, as these receptors have a direct link to the cell's intrinsic death machinery (18). TRAIL, a member of the TNF family of death-inducing ligands, is a prime candidate for cancer therapy because it almost selectively induces apoptosis in a wide spectrum of cancers with no or minimal toxicity on normal human cells. Agents triggering TRAIL receptors, such as agonistic TRAIL receptor antibodies or recombinant TRAIL, have been extensively studied in preclinical models and are currently under investigation in clinical trials. However, the potential application of TRAIL for cancer therapy is resistant to the agents. Therefore, further insights into the molecular determinants of TRAIL-induced apoptosis are pivotal to improve outcome of TRAIL treatment in NSCLC (19).

RNAi is a process of sequence-specific post-transcriptional gene silencing mediated by double-stranded RNA. Compared with conventional single-stranded antisense oligonucleotides technology, RNAi is more efficient at silencing the corresponding gene expression. At present, the main obstacle to the 

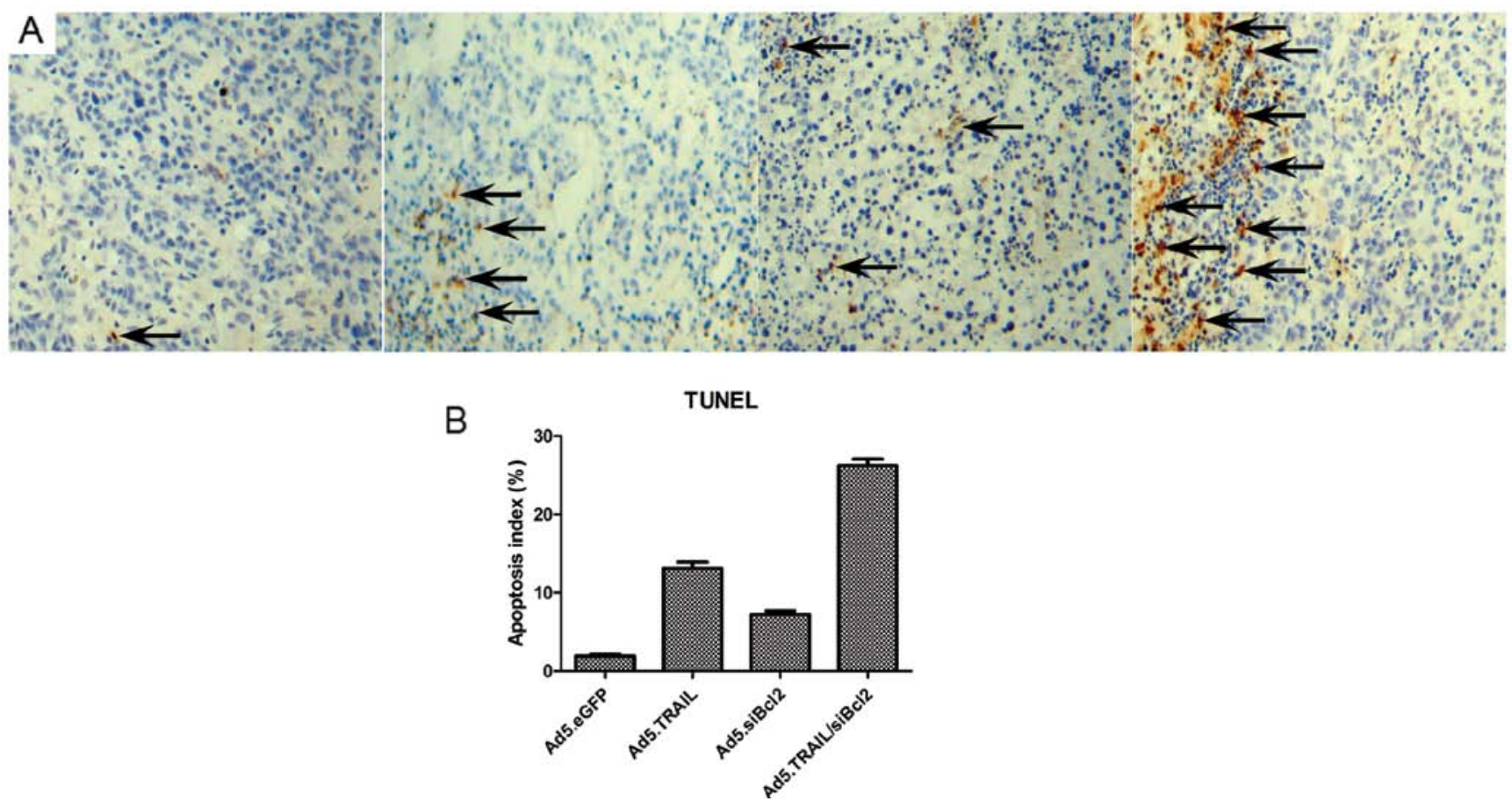

Figure 7. TUNEL staining for examination of tumor tissues after treatment with adenoviral vectors. Tumor-bearing animals were treated with Ad5.eGFP, Ad5.siBcl2, Ad5.TRAIL or Ad5.TRAIL/siBcl2. Ten days after treatment, the animals were sacrificed and the tumors were collected. The tumor sections were assayed by TUNEL. (A) Original magnification, x400. Apoptosis index of A549 xenograft tumors in nude mice treated with different viruses. (B) The apoptosis index was represented by the average percentage of TUNEL-positive cells from 10 randomly selected areas. Each bar represents the mean \pm SD ( $n=10$ ).

development of therapeutic agents using RNAi technology is a suitable delivery method. Although non-viral-based siRNA vectors are regarded as the safest delivery systems, they still constitute an essential challenge in therapeutics, limited by their poor ability to escape from the endosomal compartment and to translocate DNA into the nucleus. Another important consideration for siRNA-mediated inhibition of gene expression is whether the observed effects are free from potential IFN responses, as lipid delivery of synthetic siRNAs can reportedly induce immune activation in vivo (20). In contrast, viral vectors have been used to deliver siRNA widely and these methods tend to exhibit several advantages over nonviral vectors, such as higher transduction efficacy and more stable gene silencing. The nonreplicative viral vectors, used for siRNA delivery, can be applied to overcome these limitations (21).

In aggressive therapies, resistance to current treatment protocols constitutes a major obstacle in clinical oncology. Most anticancer agents act through induction of apoptosis in target cells and defects in apoptosis programs may confer resistance (22). Apoptosis is mediated through both the death receptor and the mitochondrial pathway. At least in some cellular systems, mitochondria also seem to be necessary for death receptor-induced apoptosis. The mitochondrial pathway of apoptosis can be blocked by the anti-apoptotic protein Bcl-2 which has been localized to cellular membranes, e.g. the mitochondrial membrane. The Bcl-2 gene is the prototype of a class of genes that contributes to neoplastic progression by enhancing tumor cell survival through the inhibition of apoptosis (23). Bcl-2 levels are elevated in follicular lymphoma, melanomas, prostate and gliomas suggesting that a decrease in Bcl-2 might sensitize cells to apoptotic stimuli (24). Bcl-2 is highly expressed in many cell lines and primary tumors including neuroblastoma or glioblastoma. High Bcl-2 expression has been correlated with poor prognosis in some cases and it is also likely to play a role in chemoresistance. Thus, novel strategies are needed to target resistance, e.g. due to Bcl-2 overexpression. As hypothesized, downregulation of Bcl-2 by siRNA sensitized NSCLC cells to Apo2L/TRAILinduced apoptosis (Fig. 4). Activation of the caspase cascade was blocked at the level of complete activation of effector caspases such as caspase-3/7 in NSCLC cell lines poorly responding to TRAIL. TRAIL stimulated cleavage of PARP following downregulation of Bcl-2 in A549 cells (Fig. 5B). Thus, downregulation of Bcl-2 may activate the mitochondrial arm (intrinsic pathway) to amplify the effects of death receptor-mediated apoptosis.

To improve the therapeutic efficiency, we combined this virotherapeutic agent with RNAi technology and conventional gene therapy method. In this study, as proposed, adenovirusmediated siRNA can efficiently silence the expression of Bcl-2 in vitro, leading to direct induction of cell death in NSCLC cell line and increase in the sensitivity of both NSCLC cell lines to Ad5.TRAIL mediated apoptosis, but with no apparent impact on normal cells (WI-38). Bcl-2 may regulate caspase activation through sequestration of unidentified caspase adaptors/ activators. Moreover, Bcl-2 could directly interact as substrate with different subsets of caspases (25). These factors might be important for A549 cells where Bcl-2 was overexpressed providing protection against Ad.TRAIL-mediated apoptosis, indicating that the caspase machinery activated by TRAIL in A549 cells might be preferentially inhibited by Bcl-2. To further prove the synergistic efficacy, our in vivo data showed that Ad5.shBcl-2 could promote antitumor activity of Ad5. TRAIL in NSCLC xenograft tumors. These phenomena 
potentially suggest that adenoviral vectors are effectively infected the NSCLC for long-term siRNA delivery.

In conclusion, our adenovirus vector has both a cell cytotoxicity ability and siRNA delivery capability. Ad5.shBcl2 could knockdown Bcl-2 expression and enhance the antitumor activity of Ad5.TRAIL both in vitro and in vivo. Our findings may have important implications in the development of new strategies in cancer therapy.

\section{Acknowledgements}

This study was carried out as part of a project financed by the grant of Shandong Tackle Key Problems in Science and Technology (2010GSF10245).

\section{References}

1. Jemal A, Siegel R, Xu J and Ward E: Cancer statistics, 2010. CA Cancer J Clin 60: 277-300, 2010.

2. Houwen L: State of the art: lung cancer in China. Ann Thorac Cardiovasc Surg 9: 147-148, 2003.

3. van DM, Karim-Kos HE, Siesling S, et al: Progress in standard of care therapy and modest survival benefits in the treatment of non-small cell lung cancer patients in the Netherlands in the last 20 years. J Thorac Oncol 7: 291-298, 2012.

4. Bareschino MA, Schettino C, Rossi A, et al: Treatment of advanced non small cell lung cancer. J Thorac Dis 3: 122-133, 2011.

5. Nair BS, Bhanderi V and Jafri SH: Current and emerging pharmacotherapies for the treatment of relapsed small cell lung cancer. Clin Med Insights Oncol 5: 223-234, 2011.

6. Ashkenazi A, Pai RC, Fong S, et al: Safety and antitumor activity of recombinant soluble Apo2 ligand. J Clin Invest 104: 155-162, 1999

7. Soria JC, Smit E, Khayat D, et al: Phase $1 \mathrm{~b}$ study of dulanermin (recombinant human Apo2L/TRAIL) in combination with paclitaxel, carboplatin, and bevacizumab in patients with advanced non-squamous non-small-cell lung cancer. J Clin Oncol 28: $1527-1533,2010$.

8. Pan G, O RK, Chinnaiyan AM, et al: The receptor for the cytotoxic ligand TRAIL. Science 276: 111-113, 1997.

9. Walczak H, Degli-Esposti MA, Johnson RS, et al: TRAIL-R2: a novel apoptosis-mediating receptor for TRAIL. EMBO J 16: 5386-5397, 1997
10. Marsters SA, Sheridan JP, Pitti RM, et al: A novel receptor for Apo2L/TRAIL contains a truncated death domain. Curr Biol 7: 1003-1006, 1997.

11. Emery JG, McDonnell P, Burke MB, et al: Osteoprotegerin is a receptor for the cytotoxic ligand TRAIL. J Biol Chem 273: 14363-14367, 1998

12. Zhang Y, Wang JH, Lu Q and Wang YJ: Bag3 promotes resistance to apoptosis through $\mathrm{Bcl}-2$ family members in non-small cell lung cancer. Oncol Rep 1: 109-113, 2011.

13. Kroemer G: The proto-oncogene $\mathrm{Bcl}-2$ and its role in regulating apoptosis. Nat Med 3: 614-620, 1997.

14. Griffith TS and Lynch DH: TRAIL: a molecule with multiple receptors and control mechanisms. Curr Opin Immunol 10: 559-563, 1998.

15. Carr LL, Finigan JH and Kern JA: Evaluation and treatment of patients with non-small cell lung cancer. Med Clin North Am 95: 1041-1054, 2011.

16. Daly BD, Cerfolio RJ and Krasna MJ: Role of surgery following induction therapy for stage III non-small cell lung cancer. Surg Oncol Clin N Am 20: 721-732, 2011.

17. Coleman $\mathrm{MH}$ and Bueno R: Role of adjuvant chemotherapy in NSCLC (stages I to III). Surg Oncol Clin N Am 20: 757-767, 2011.

18. Ashkenazi A: Targeting death and decoy receptors of the tumournecrosis factor superfamily. Nat Rev Cancer 2: 420-430, 2002.

19. McGrath EE: The tumor necrosis factor-related apoptosisinducing ligand and lung cancer: still following the right TRAIL? J Thorac Oncol 6: 983-987, 2011.

20. Bridge AJ, Pebernard S, Ducraux A, Nicoulaz AL and Iggo R: Induction of an interferon response by RNAi vectors in mammalian cells. Nat Genet 34: 263-264, 2003.

21. Carette JE, Overmeer RM, Schagen FH, et al: Conditionally replicating adenoviruses expressing short hairpin RNAs silence the expression of a target gene in cancer cells. Cancer Res 64: 2663-2667, 2004.

22. Kaufmann SH and Earnshaw WC: Induction of apoptosis by cancer chemotherapy. Exp Cell Res 256: 42-49, 2000.

23. Gross A, McDonnell JM and Korsmeyer SJ: Bcl-2 family members and the mitochondria in apoptosis. Genes Dev 13: 1899-1911, 1999.

24. Jansen B and Zangemeister-Wittke U: Antisense therapy for cancer - the time of truth. Lancet Oncol 3: 672-683, 2002.

25. Cory S and Adams JM: The Bcl-2 family: regulators of the cellular life-or-death switch. Nat Rev Cancer 2: 647-656, 2002. 